\title{
Load Shedding Strategy Application Using Fuzzy Logic
}

\author{
Moez Ben Hessine \\ Latice Laboratory at High School of Sciences and Techniques \\ University of Tunis, ESSTT, 5 Av Taha Hussien 1008 \\ Tunisia \\ benhessinemoez@yahoo.fr
}

\author{
Houda Jouini \\ Latice Laboratory at High School of Sciences and Techniques \\ University of Tunis, ESSTT, 5 Av Taha Hussien 1008 \\ Tunisia \\ houda.jouini@gmail.com
}

\author{
Souad Chebbi \\ Latice Laboratory at High School of Sciences and Techniques \\ University of Tunis, ESSTT, 5 Av Taha Hussien 1008 Tunisia \\ souad.chebbi@yahoo.com
}

\begin{abstract}
As a perspective to ensure the voltage and frequency electrical network stability, the load shedding constitutes a desirable action to maintain the network service quality and to control its vulnerability. In this paper, we propose a new intelligent load shedding strategy applying fuzzy control algorithms. This strategy is based on the estimate, in real time, of the load quantity to shed. Calculation algorithms containing fuzzy controllers generate command vectors ensuring the load shedding of a pre calculated proportion loads in order to reestablish the power balance and to lead the electrical network to a new stable condition.
\end{abstract}

Index Terms - Load shedding, Fuzzy logic, PSRF, NSRF, Sensitivity, positive sequence, negative sequence, Eigen value.

\section{INTRODUCTION}

Various disturbances occur in electrical networks every year. They lead to blackouts. As the frequency and voltage represent two important parameters for the power system safety, it should have a continuous control of these parameters and this to ensure the best service quality. It's characterized by standards criterions related to the service continuity to the voltage profile, to the purity of injected frequency, to the network static and transient robustness opposite a set of possible exploitations and disturbances scenarios. The networks vulnerability control is an important rivalry, since preventives and curatives means can be considered in order to guarantee networks service quality. In the case of vulnerable cascading events leading to blackouts, the load shedding will be the most desirable action avoiding network instability.

Different methods $[1, ., 9]$ was proposed in order to decide the place and the quantity of loads to be shed. In this context we develop, in the present paper, a new load shedding strategy based on the fuzzy logic named intelligent load shedding.

\section{PUT IN SITUATION}

In disturbed mode, the direct and quadrature components of variables $\vec{X}$ (currents or voltage) at different buses will be affected by oscillation terms that we propose to identify [9]. Thus, we exploit two study reference frames: a positive synchronous reference frame (PSRF) moving with the pulsation $+\omega$, or negative synchronous reference frame (NSRF) $-\omega$ (Fig. 1).

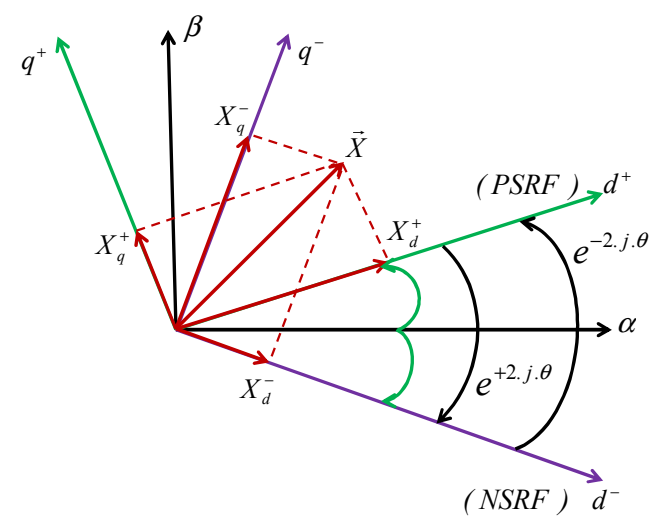

Fig. 1.Study references 
We write then:

$$
\left\{\begin{array}{l}
\bar{X}_{\mathrm{dq}}^{+}=\bar{X}_{\mathrm{dqp}}^{+}+\bar{X}_{\mathrm{dqn}}^{+} \\
\bar{X}_{\mathrm{dq}}^{-}=\bar{X}_{\mathrm{dqp}}^{-}+\bar{X}_{\mathrm{dqn}}^{-}
\end{array}\right.
$$

Compared to positive synchronous reference frame (PSRF), we express the (d, q) axe components of an electric variable $\bar{X}$ as follow:

$$
\left\{\begin{array}{l}
X_{d}^{+}=X_{d p}^{+}+X_{d n}^{-} \cdot \cos (2 \cdot \theta)+X_{q n}^{-} \cdot \sin (2 \cdot \theta) \\
X_{q}^{+}=X_{q p}^{+}-X_{d n}^{-} \cdot \sin (2 \cdot \theta)+X_{q n}^{-} \cdot \cos (2 \cdot \theta)
\end{array}\right.
$$

Thus, during the appearance fault phase, any electric quantity will be considered as the sum of a continuous component $\bar{X}_{0}$ which comes to superimpose a fluctuating component $\bar{X}_{2 f}$ with double network frequency 2. $f$. It comes then:

$$
\bar{X}=\bar{X}_{0}+\bar{X}_{2 f}
$$

On the basis of the apparent power expression injected at bus $j$ :

$$
\bar{S}_{j}=\bar{V}_{j} \cdot \bar{I}_{j}^{*}
$$

(4) is translated in the positive synchronous reference frame (PSRF) by:

$$
\left.\bar{S}_{j}=\left[V_{j d}^{+}+j \cdot V_{j q}^{+}\right]\right] \cdot\left(\begin{array}{c}
\left(R Y_{j k}^{s h}-j \cdot I Y_{j k}^{s h}\right) \cdot\left(V_{j d}^{+}-j \cdot V_{j q}^{+}\right) \\
+\left(R Y_{j k}^{L}-j \cdot I Y_{j k}^{L}\right) \cdot\left(V_{j d}^{+}-j \cdot V_{j q}^{+}\right)
\end{array}\right)
$$

$V_{j d}^{+}, V_{j q}^{+}$: direct and quadrature components of the voltage at bus $j$ in the PSRF reference frame). $R Y_{j k}^{s h}$ and $I Y_{j k}^{s h:}$ respectively real and imaginary parts of the shunt admittance of line.

The active and reactive injected powers at bus $j$ express by:

$$
\left\{\begin{aligned}
P_{j}= & V_{j d}^{+}\left[R Y_{j k}^{s h} \cdot V_{j d}^{+}-I Y_{j k}^{s h} \cdot V_{j q}^{+}+R Y_{j k}^{L} \cdot V_{j d}^{+}-I Y_{j k}^{L} \cdot V_{j q}^{+}\right] \\
& +V_{j q}^{+}\left[R Y_{j k}^{s h} \cdot V_{j q}^{+}+I Y_{j k}^{s h} \cdot V_{j d}^{+}+R Y_{j k}^{L} \cdot V_{j q}^{+}+I Y_{j k}^{L} \cdot V_{j d}^{+}\right] \\
Q_{j}= & V_{j q}^{+}\left[R Y_{j k}^{s h} \cdot V_{j d}^{+}-I Y_{j k}^{s h} \cdot V_{j q}^{+}+R Y_{j k}^{L} \cdot V_{j d}^{+}-I Y_{j k}^{L} \cdot V_{j q}^{+}\right] \\
& -V_{j d}^{+}\left[R Y_{j k}^{s h} \cdot V_{j q}^{+}+I Y_{j k}^{s h} \cdot V_{j d}^{+}+R Y_{j k}^{L} \cdot V_{j q}^{+}+I Y_{j k}^{L} \cdot V_{j d}^{+}\right]
\end{aligned}\right.
$$

Taking into account of (6), the active and reactive power expressions (7) are put in the form:

$$
\left\{\begin{array}{l}
P_{j}=P_{o}+P_{2 f} \\
Q_{j}=Q_{o}+Q_{2 f}
\end{array}\right.
$$

with: $P_{o}$ and $Q_{o}$ indicate functions of $(\mathrm{d}, \mathrm{q})$ axe components of current and voltage positive sequences $\left(X_{d p}^{+}, X_{q p}^{+}\right)$at the same bus. $P_{2 f}$ and $Q_{2 f}$ are functions of (d, q) axe component of the current and voltage negative sequences $\left(X_{d n}^{-}, X_{q n}^{-}\right)$at the same bus.

The fluctuations which appear in the injected powers at different buses show the possibility for serious difficulties with which clash the algorithms treating these variables to stabilize the power studied system.

We develop a new load shedding strategy containing fuzzy controllers, aiming at stabilizing an electrical network during the fault appearance phase, taking into account the fluctuations of different electric variables.

\section{INTELLIGENT LOAD SHEDDING STRATEGY}

Various service quality control strategies of electrical network were proposed, certain are qualified as preventive ones whereas others are qualified as curative [4]. By mean of the equation system (7), we implemented a filtering technique ensuring the fluctuating component evaluation of the injected powers at different producing buses. Thus, we used cuts band filters centered on a double cut-off frequency of the studied network. This technique ensures the rejection of the fluctuating components contained in positive and negative sequences presented by Fig. 2 . Consequently, we manage to reconstitute components similar to those of a balanced mode.

Nevertheless, this filtering technique does not guarantee the injected power amplitudes. For that reason, we estimated the fluctuating power amplitudes and added them to those continuous components (Fig. 2) in order to quantify the power quantity to shed.

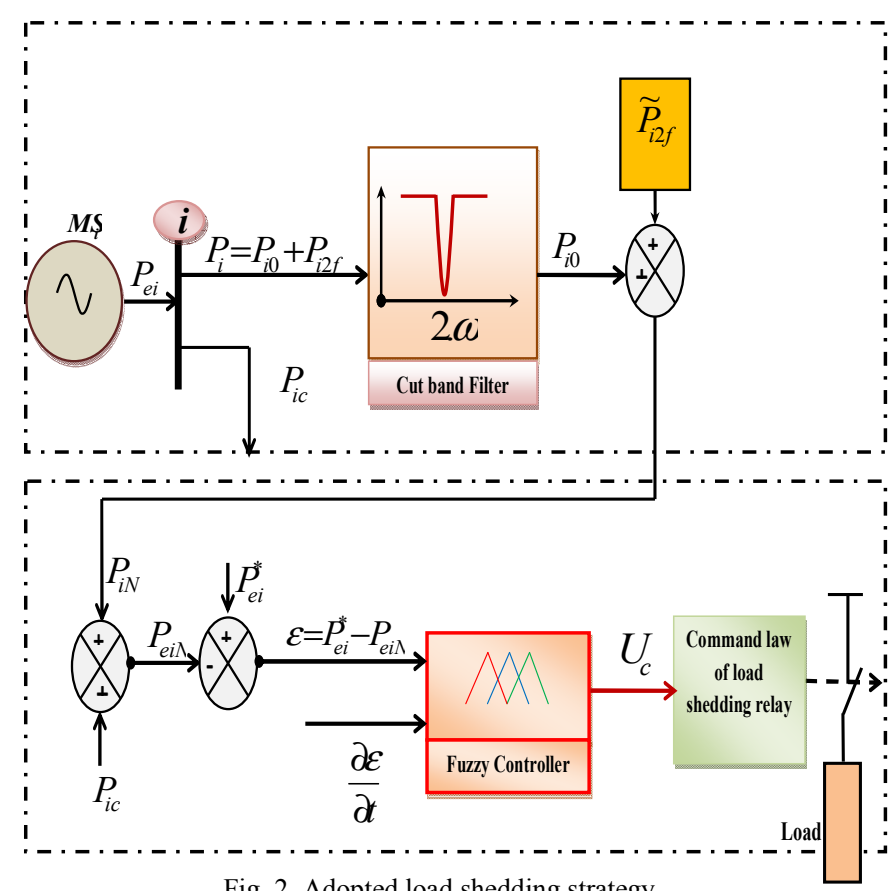

Fig. 2. Adopted load shedding strategy. 
This control strategy consists on evaluating, in real time, the load quantity to be shed. This load shedding strategy was based on the fuzzy controller installation at each producing bus (Fig. 2).

This controller treated two inputs $\left(\varepsilon, \frac{\partial \varepsilon}{\partial t}\right)$, the first input is materialized by the gap between the power generated amplitudes by the machine given by (8) and those of references required by the network $P_{e i}^{*} \mathrm{i}=\{1, \ldots$, machines $\}$ before and after the fault appearance phase $\varepsilon_{i}=\left(P_{e i}^{*}-P_{e i N}\right)$. The second input represents the variation of this gap compared to time. The make decision of the fuzzy controllers represents the load quantity to be shed at different buses according to their sensitivity degrees.

$$
\left\{\begin{array}{l}
P_{e i N}=P_{i N}+P_{i C} \\
P_{i N}=P_{i 0}+\widetilde{P}_{i 2 f}
\end{array}\right.
$$

With:

$P_{i 0}$ : represent the continuous power quantity,

$\widetilde{P}_{i 2 f}$ : represent the fluctuating power quantity amplitude,

$P_{i C}:$ represent the consumption quantity at bus $\mathrm{i}$,

$U_{C}$ : represent the total load quantity to shed at different buses. Then :

$$
U_{C i}=U_{C i-1}+U_{C i-2}+\ldots U_{C i-j} \ldots+U_{C i-b u s e s}
$$

We must insist on the fact that the load quantities to be shed $P_{d j}$, at bus $j$, is indeed the resultant of the elementary command vectors $U_{C i-j}$ generated by the various fuzzy controllers, affected to different machines (Nmachines) as follow:

$$
U_{C j}=\sum_{i=1}^{i=\text { Nmachines }} U_{C n-j}
$$

The load quantities to be shed $P_{d j}$ at bus $j$, is established in accordance with (11).

$$
P_{d j}=\sum_{i=1}^{i=N m} \frac{\frac{\partial P_{e i}}{\partial P_{j}}}{\sum_{k=1}^{k=\text { buses }} \frac{\partial P_{e i}}{\partial P_{k}}} \cdot U_{C i}
$$

With:

$\frac{\partial P_{e i}}{\partial P_{j}}$ : constitute indicators of the sensitivity degree of machine $\mathrm{i}$ to the power injected at different buses of the network $P_{d j}$ : load quantity to shed at each bus. In order to allow more precision to the action brought by the integrated fuzzy controllers and to take into account the maximum of states suitable to affect the different variables to be treated, we defined nine membership functions for the two input variables: NVL (Negative Very Large), NL (Negative Large), Nm (Negative Medium), NS (Negative Small), ZE (Zero), PS (Positive Small), PM (Positive Medium), PL (Positive Large) and PVL (Positive Very Large). Thus we formulated 81 fuzzy rules which manage the command vector (Table 1).

TABLE 1

DIFFERENT FUZZY RULES TO MANAGE THE COMMAND VECTOR

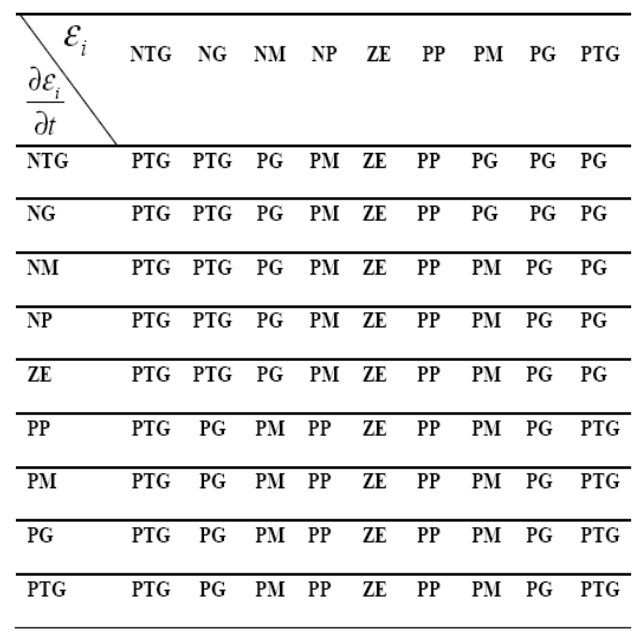

The following paragraph will be reserved for the study criterion development of the electrical network stability in the presence of a disturbance.

\section{STUDY CRITERION OF THE STUDIED NETWORK STABILITY}

Considering the electrical network is very complex and in order to study its dynamic stability, with our point of view, we will not be able to apply easily various criteria. This is why we had resorts to the Eigen value analysis of the studied system state model [10]. For our case, we considered 4 dynamic variables for each machine integrated in the network definite by $\left[\delta_{i}, \omega_{i}, E_{q i}^{\prime}, E_{d i}^{\prime}\right]$ and two algebraic variables $\left[V_{i}, \alpha_{i}\right]$; what makes it possible to define a state vector $\left[X_{i}\right]$ of and a command vector characterized by:

$$
\left\{\begin{array}{l}
{\left[X_{i}\right]=\left[\delta_{i}, \omega_{i}, E_{q i}^{\prime}, E_{d i}^{\prime}\right]^{T}} \\
{\left[Y_{i}\right]=\left[V_{i}, \alpha_{i}\right]^{T}}
\end{array}\right.
$$

The $\left[X_{i}\right]$ determination is possible only by means of the following system resolution:

$$
\left\{\begin{array}{l}
{\left[\dot{X}_{i}\right]=F_{i}\left(X_{i}, Y_{i}\right)} \\
{[0]=G_{i}\left(X_{i}, Y_{i}\right)}
\end{array}\right.
$$


With:

$F_{i}\left(X_{i}, Y_{i}\right)$ represent the dynamic model of the $\mathrm{i}^{\text {th }}$ machine and $H_{i}\left(X_{i}, Y_{i}\right)$ represent the energy balance. In other words, we have to make with the following system:

$$
\left\{\begin{array}{l}
\Delta\left[\dot{X}_{i}\right]=\left[A_{1 i}\right] \cdot \Delta\left[X_{i}\right]+\left[B_{1 i}\right] \cdot \Delta\left[Y_{i}\right] \\
{[0]=\left[A_{2 i}\right] \cdot \Delta\left[X_{i}\right]+\left[B_{2 i}\right] \cdot \Delta\left[Y_{i}\right]}
\end{array}\right.
$$

The matrices, $\left[A_{1 i}\right],\left[B_{1 i}\right],\left[A_{2 i}\right]$ and $\left[B_{2 i}\right]$ have respectively dimensions [4×4], $[4 \times 2],[2 \times 4]$ and $[2 \times 2]$, their coefficients can be deduced without particular difficulty starting from the following partial derivative:

$$
\left\{\begin{array}{l}
{\left[A_{1 i}\right]=\left[\frac{\partial F_{i}}{\partial X_{i}}\right] ;\left[B_{1 i}\right]=\left[\frac{\partial F_{i}}{\partial X_{i}}\right]} \\
{\left[A_{2 i}\right]=\left[\frac{\partial G_{i}}{\partial X_{i}}\right] ;\left[B_{2 i}\right]=\left[\frac{\partial G_{i}}{\partial X_{i}}\right]}
\end{array}\right.
$$

By replacing the value of $\Delta\left[Y_{i}\right]$ drawn from the second relation of equation systems in the first relation (14) of the same system, we find:

$$
\Delta\left[\dot{X}_{i}\right]=\left[A_{i}\right] \cdot \Delta\left[X_{i}\right]
$$

According to the signs of the Eigen value real parts $\lambda_{k i}, \mathrm{k}=\{1, \ldots, 4\}$ of the matrix $\left[A_{i}\right],[4 \times 4]$ dimensions, we come to a conclusion about the studied machine-network stability.

\section{SimUlations AND RESUlTS}

The study model is introduced by a typical system containing the IEEE test 14 buses network (Fig. 3).

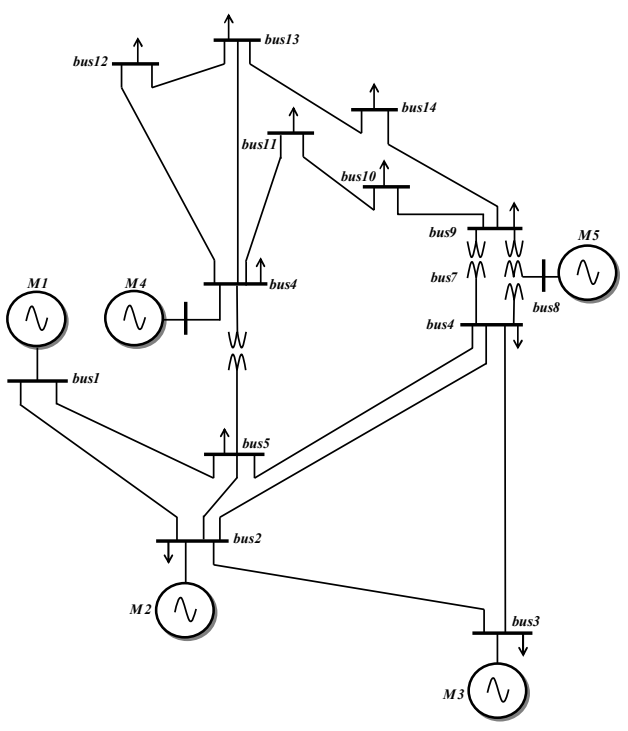

Fig. 3. IEEE 14 bus test network
In order to quantify the contribution of the implemented intelligent control strategy, we simulated a fault scenario. Simulations were carried out on MATLAB 2008 software.

We simulated the studied system behavior at the fault time characterized by the generator outage at $t=65 \mathrm{~s}$. In absence of the adopted load shedding strategy, the voltage level at different buses undergoes a notable degradation leading to a phenomenon of total voltage collapse (Fig. 4).

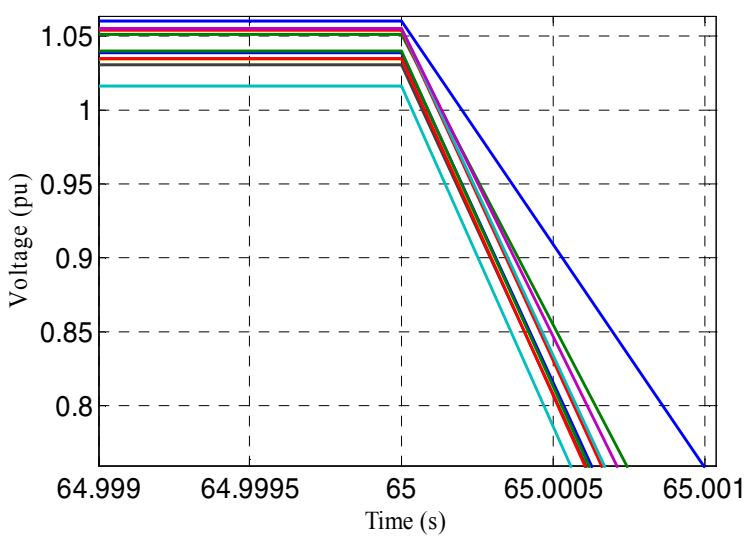

Fig. 4. Voltage level in the event of generator outage in the presence of the load shedding strategy.

In the second phase of simulation, we simulated the studied system behavior at the fault time characterized by the generator outage. By integration of the new control strategy, we gave the voltages at different buses in new steady states and this during the fault appearance phase.

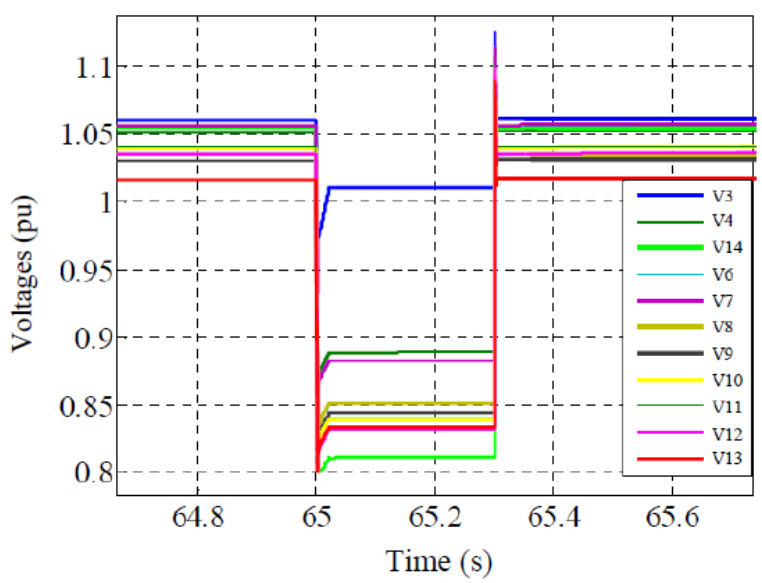

Fig. 5.Voltage levels in the generator outage event in the presence of the load shedding strategy.

As for the frequential behaviors at different machines, it proves that these last keep a perfect synchronization with the connection network during the fault appearance phase owing to the fact that the amplitudes of their fluctuating frequencies $f_{f}$ limited by tolerable extremums, Fig. 6. 


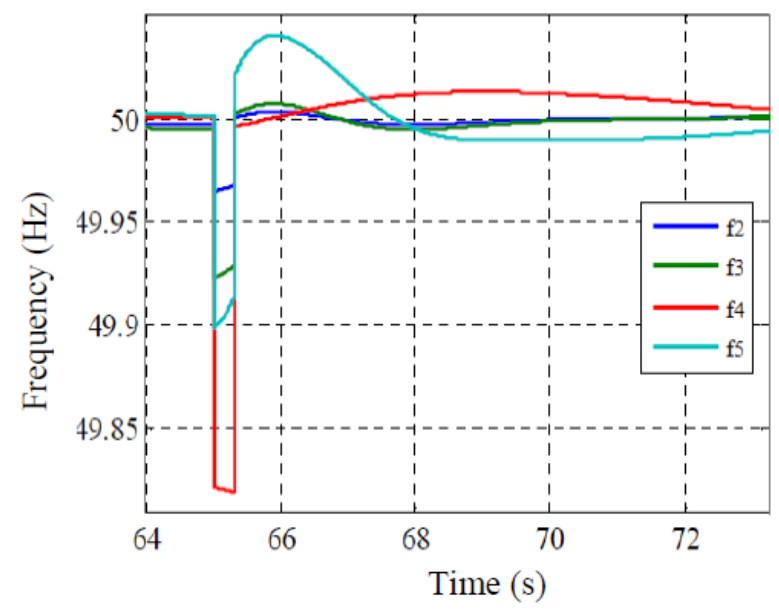

Fig. 6. Frequency levels in the event of generator outage in the presence of the load shedding strategy.

The temporal evolutions of the real parts of the Eigen values of the state matrix $\left[A_{2}\right]$, relating to the machine $G_{2}$, comes to more justify the potential performances of the adopted stabilization strategy in this fault case mentioned above to the generator outage (Fig. 7).

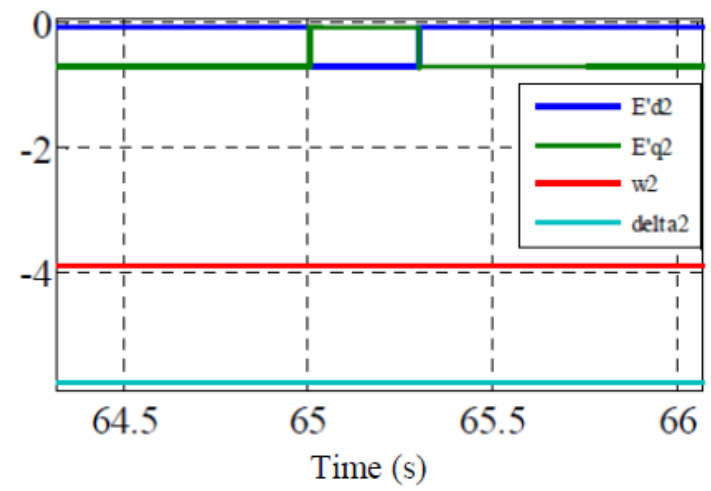

Fig. 7. Temporal evolution of the eigen value real parts machine (60 MVA), cases of generator outage.

\section{CONCLUSION}

We proposed in this paper a strategy allowing ensuring network service continuity in the presence of situations described as vulnerable.
This strategy founded on a perfect recognition of the variation of the powers delivered by the machines compared to values of reference evaluated in normal functioning mode. The treated fault proves the strategy potentiality.

\section{REFERENCES}

[1] Souad Chebbi, Houda Jouini, Mohamed Annabi, "Application of SIME Method to Tunisian Nework" International Conference on Electric Power and Energy Conversion Systems, EPECS, IEEE XPLORE,2009.

[2] Roberto Faranda, "load shedding: New Proposal" IEEE transactions on power systems, vol.22.no.4, November 2007.

[3] D. K. SUBRAMANIAN,"Optimum load shedding through Programming techniques", IEEE transactions on power apparatus and systems, vol. pas-90, no. 1, January/February 1971

[4] Tomaz Tomasic, Gregor Verbic, Ferdinand Gubina"revision of the underfrequency load-shedding sheme of the Slovinian power system" IEEE ,2005.

[5] C.J.Parker, I.F.Morrison, D.Sutanto,"'Simulation of load shedding as a corrective action against votage colapse",Electrical Power System Research, Elsevier 22. pp 235-241,1998.

[6] AhmedM.A.Haidar,Asah Mohamed,Ani Hussain,"Vulnerability control of large scale interconnected power system using neurofuzzy load shedding approach",Expert Systems With Applicatons, Elsevier 37.pp. 3171-3176, 2010.

[7] Adly A.Girgis, Shruti Marthure,"application of active power sensitivity to frequency and voltage variations on load shedding",Electric Power Systems Reseearch, Elsevier 80 .pp. 306-310,2010.

[8] A.N.Udupa, G.K.Purushothama, .Parthasarathy,D.Thukaram,"A fuzzy control for network overload alleviation", Electrical Power and Energy System,Elsevier 123.pp. 119-128,2001.

[9] Moez Ben Hessine,Sahbi Marrouchi,Souad Chebbi, Houda Jouini, Rabeh Abbessi, International Review of Modeling and Simulations (IREMOS)-December2011; «Preventive and Curative Strategies Based on Fuzzy Logic Used for Voltage Stabilization of an Electrical Network» ISSN 1974-9821 Vol. 4 N.6 . December2011

[10] Moez Ben Hessine, Houda Jouini, Souad Chebbi and Sahbi Marrouchi, International Review of Electrical and Engineering (IREE)-October 2012; « Voltage and Frequency Stabilization of Electrical Networks by Using load Shedding Strategy Based on Fuzzy Logic Controllers ». 